\title{
The Presence of a Revolution Load and Its Rotational Speed of Half the Speed of the Journal Are Necessary Conditions for the Half-Frequency Whirl of Radial Bearings
}

\author{
Jingyuan $\mathrm{Li}^{1}$ \& Zifeng $\mathrm{Li}^{1}$ \\ ${ }^{1}$ Petroleum Engineering Department, Yanshan University, Qinhuangdao 066004, Hebei, China \\ Correspondence: Zifeng Li, Petroleum Engineering Department, Yanshan University, Qinhuangdao 066004, \\ Hebei, China. E-mail: zfli@ysu.edu.cn
}

Received: January 22, 2016 Accepted: February 1, 2016 Online Published: February 15, 2016

doi:10.5539/apr.v8n2p16

URL: http://dx.doi.org/10.5539/apr.v8n2p16

\begin{abstract}
In the radial sliding bearing lubrication theory, the half-frequency whirl phenomena are commonly introduced. However, the correlation of the half-frequency whirl theory with the measured resonance phenomenon is low. This paper studies the establishment of the half-frequency whirl theory, and finds that while researchers are aware of the half-frequency whirl, they disregard the necessary conditions of its occurrence. If there is no revolution load, then the half-frequency whirl does not comply with the principle of minimum potential energy. If it does not conform to the principle of minimum energy dissipation, then the half-frequency whirl does not exist. Only when the revolution load exists and its speed is half the speed of the journal will the half frequency whirl occur. Engineering oil whip results from the vibration of the rotating system. A precise radial sliding bearing lubrication theory should use cylindrical coordinates, consider the compressibility of the liquid, and consider the surface shear stress in the journal bearing capacity calculations.
\end{abstract}

Keywords: revolution load, rotational speed, whirl, radial bearings

\section{Introduction}

In the radial sliding bearing lubrication theory, the half-frequency whirl phenomenon is commonly introduced (Newkirk, 1924; Newkirk \& Taylor, 1925; Yao, 1991; Yao, 1992; Wen \& Huang, 2008; Zhang et al., 2008). The traditional half-frequency whirl, or half-speed whirl, is defined as the rotation of the journal around the center of the bearing while the journal rotates around itself; its frequency is approximately half the journal rotational frequency. As long as the journal rotates, the vortex of the journal around the center of the bearing will occur, and the vortex frequency is half as much as that of the shaft neck rotation; this has nothing to do with the bearing load. The correlation of the traditional half-frequency whirl to the measured rotor resonance phenomenon is low (Yao, 1991; Yao, 1992). It is important to explore the root causes for this low correlation.

This study finds that the traditional definition of the half-frequency whirl is imprecise and is one of the root causes of the inconsistency of the measured rotor resonance phenomenon with the definition. The half-frequency whirl should be redefined as the journal center whirl radius reaching a maximum for a radial sliding bearing while the rotating speed of the load is half the rotating speed of the journal.

\section{The Traditional Half-Frequency Whirl Definition and the Predicted Journal Center Position in the Traditional Lubrication Theory are far from the Actual Measured Position}

Figure 1 shows the journal position of a bearing uniformly rotating clockwise under a constant load $W$. The journal center $O_{1}$ is at the lower left of the bearing center $O$.

(1) According to the traditional half-frequency whirl definition, the center of the journal $O_{1}$ will rotate around the center of the bearing $O$ with a clockwise angular speed of $\omega / 2$. This is clearly inconsistent with the facts. Previous experimental studies do not fully support the traditional half-frequency whirl definition (Yao, 1991; Yao, 1992).

(2) According to the principle of minimum potential energy, the journal center $O_{1}$ can only be at the lower left of the bearing center $O$, and cannot whirl around the bearing center. 
(3) According to the principle of minimum energy dissipation (Zhou \& Tang, 2007), the journal center $O_{1}$ can only be at the lower left of the bearing center $O$, and cannot whirl around the bearing center.

(4) According to the radial sliding bearing lubrication theory (Wen \& Huang, 2008), the center of the journal $O_{1}$ should be located on the horizontal line through the bearing center $O$, e.g., $\mathrm{O}_{2}$ with the journal outline being in the dotted circle. This is clearly inconsistent with the actual experimental results.

Therefore, the half-frequency whirl phenomenon should not exist in this condition. Engineering oil whip (Yao, 1991; Yao, 1992) should result from the vibration (including the resonance) of the rotating system (Dupont, 2003; Kalita \& Kakoty, 2004; Castro et al., 2008).

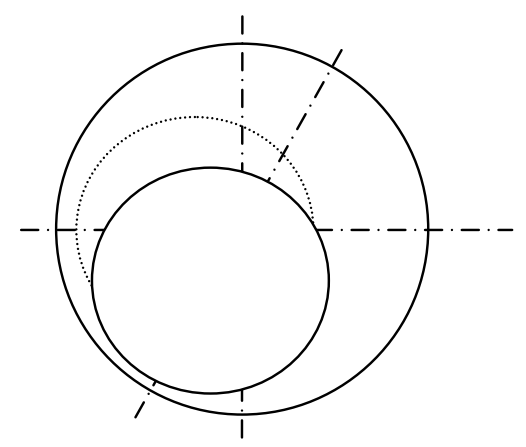

Figure 1. The actual and theoretical locations of the journal

\section{Mathematical Derivation of the Half- Frequency Whirl}

There are two methods to derive the half-frequency whirl theory: the volume conservation method $[3,6]$ and the dynamic load bearing method (Wen \& Huang, 2008).

\subsection{Volume Conservation Method}

Figure 2 is a journal whirl analysis. $O$ is the bearing center, $O_{1}$ is the journal center, $R$ is the journal radius, $c$ is the average bearing clearance, $e$ is the eccentricity, $\omega$ is the rotational angular velocity of the journal, and $\Omega$ is the revolution angular velocity of the journal. According to the continuous flow theory (Zhang et al., 2008):

$$
\frac{1}{2}(\omega R-\Omega e)(c+e)-\frac{1}{2}(\omega R+\Omega e)(c-e)=2 e \Omega R
$$

In ref (Zhang et al., 2008), the right-hand side of formula (1) is $2 \Omega R$ : a suspected printing error.

From formula (1), the whirl angular velocity of the journal center is

$$
\boldsymbol{\Omega}=\omega /(2+c / R)
$$

In formula (2), when $c / R$ is insignificant, then $\Omega=\omega / 2$, i.e., the whirl angular velocity is half the rotational angular velocity in the same direction.

The theory is that there is a half-frequency whirl as long as the journal rotates, which is clearly inconsistent with the facts.

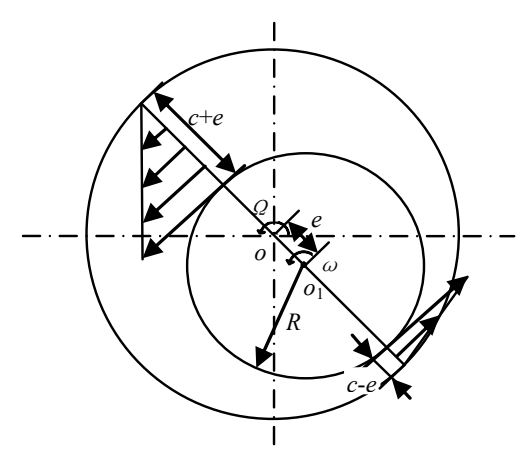

Figure 2. Journal whirl analysis (Zhang et al., 2008) 


\subsection{Dynamic Load Bearing Method}

Wen and Huang (2008) derives the carrying capacity of the bearing and its journal in both rotation and revolution (Figure 3):

$$
\left.\begin{array}{l}
\frac{S}{12 \pi^{2}}=\frac{\varepsilon}{\left(2+\varepsilon^{2}\right)\left(1-\varepsilon^{2}\right)^{1 / 2}}\left(1-2 \frac{\Omega}{\omega}\right) \\
\psi=\frac{\pi}{2}
\end{array}\right\}
$$

where $S=\frac{P}{N \eta}\left(\frac{C}{R}\right)^{2}, P=\frac{W}{L D}, N$ is the journal speed $(\mathrm{r} / \mathrm{s}), L$ is the bearing length, $D$ is the journal diameter,

$\eta$ is the liquid viscosity, $\varepsilon$ is the eccentricity $=e / c, W$ is the load, and $\psi$ is the load position angle.

The carrying capacity in formula (3) is composed of two components, a specified load when the journal rotates with angular velocity $\omega$; and the load when the journal does not rotate but the load rotates with angular velocity $\Omega$. Both have a phase difference of $180^{\circ}$. The total carrying capacity of formula (3) can be calculated by the algebraic sum.

The formula also shows that the carrying capacity of the rotational load bearing depends on the relative values of $\omega$ and $\Omega$. When $\Omega=0$, the condition represents a stable load bearing. When $\Omega=\omega / 2, S$ is zero, indicating that there is a sharp vibration of the half-frequency whirl in the bearing.

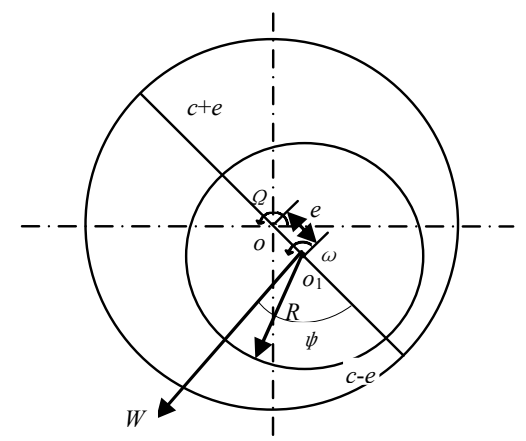

Figure 3. Shaft neck moment analysis under a dynamic load in a radial sliding bearing (Wen \& Huang, 2008)

\section{Model Error and the Unreasonable Simplification of the Radial Sliding Bearing Lubrication Theory}

The Newtonian Viscous Force Formula Is Not Suitable for Solving the Rotational Flow

The Newtonian viscous force formula is the simplest formula in fluid mechanics:

$$
F=A \eta \frac{\mathrm{d} U}{\mathrm{~d} Z}
$$

where $F$ is the viscous force, $A$ is the area, $U$ is the liquid velocity, and $z$ is the height.

Formula (4) can only be used to solve the problem of parallel laminar flow and cannot be used to solve rotating flow (Liu et al., 2005) without inducing large errors.

The radial sliding bearing lubrication theory uses formula (4) to establish formula (3) and approximately calculate the rotational flow field between the journal and the bearing. This results in a large error.

\subsection{The Compressibility of the Liquid Should be Considered}

The classic theory of lubrication assumes an incompressible liquid for ease of calculation, resulting in the center of the journal center and the center of the bearing remaining on the same horizontal line. Liquid compressibility should be considered in the lubrication theory. 
4.2 Flow Volume is Oversimplified in the Volume Conservation Method for the Derivation of the Half-Frequency Whirl

For the eccentricity condition shown in Figure 2, the journal drives fluid flow as it rotates. There are carrying liquid flows and pressure flows. In the volumetric conservation method for the derivation of the half-frequency whirl, only the carrying liquid flow is considered, and it is approximated. Figure 4 uses the simplest concentric question to illustrate that the carrying liquid flow in the volume conservation method is approximate.

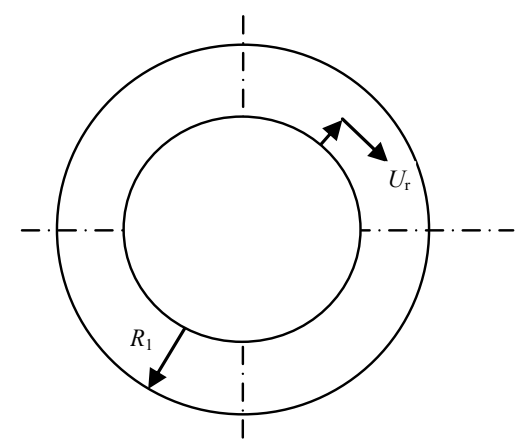

Figure 4. The journal rotating in the center of the bearing

In Figure 4, $r$ is the radial coordinate, $R_{1}$ is the bearing radius, $R_{2}$ is the journal radius, and $U_{\mathrm{r}}$ is the liquid tangential velocity. The carrying liquid velocity as a result of journal rotation is determined by the following method (Li et al., 1995; Li, 2008):

$$
\begin{gathered}
\frac{\mathrm{d}^{2} U_{\mathrm{r}}}{\mathrm{d} r^{2}}+\frac{1}{r} \frac{\mathrm{d} U_{\mathrm{r}}}{\mathrm{d} r}-\frac{U_{\mathrm{r}}}{r^{2}}=0 \\
U_{\mathrm{r}}=C_{1} r+\frac{C_{2}}{r}
\end{gathered}
$$

Given the boundary conditions $\left.U_{\mathrm{r}}\right|_{r=R_{1}}=0$ and $\left.U_{\mathrm{r}}\right|_{r=R_{2}}=R_{2} \omega$, the liquid tangential velocity is

$$
U_{\mathrm{r}}=\frac{\omega R_{2}^{2}}{R_{2}^{2}-R_{1}^{2}}\left(r-\frac{R_{1}^{2}}{r}\right)
$$

The carrying liquid flow rate is

$$
\begin{aligned}
& q_{X r}=\int_{\mathrm{R}_{2}}^{\mathrm{R}_{1}} U_{\mathrm{r}} \mathrm{d} r=\int_{\mathrm{R}_{2}}^{\mathrm{R}_{1}} \frac{\omega R_{2}^{2}}{R_{2}^{2}-R_{1}^{2}}\left(r-\frac{R_{1}^{2}}{r}\right) \mathrm{d} r \\
& =\frac{\omega R_{2}^{2}}{R_{2}^{2}-R_{1}^{2}}\left[\frac{R_{1}^{2}-R_{2}^{2}}{2}-R_{1}^{2} \ln \left(\frac{R_{1}}{R_{2}}\right)\right]
\end{aligned}
$$

rather than $R_{2} \omega \frac{h}{2}$.

Furthermore, at $\theta=0$ and $\pi, \frac{\partial p}{\partial \theta} \neq 0$, there is a pressure flow that should be acknowledged.

Oversimplification results in the fallacy of, "as long as there is journal rotation, there is a half-frequency whirl".

\subsection{The Shear Stress is not Considered in the Carrying Capacity Calculation}

There is shear stress and pressure on the journal surface. Existing theories only consider the pressure considering the shear stress. 


\section{Revolution Load and Journal Whirl}

From the experiments, the minimum potential energy principle, the principle of minimum energy dissipation (Zhou \& Tang, 2007) and the dynamic load bearing lubrication theory (Wen \& Huang, 2008), the following conclusions can be drawn:

1) If there is no revolution load, there is no journal whirl.

2) As long as there is a revolution load, there is a journal whirl.

3) When the load is a constant, its rotational speed is half the speed of the journal, and its rotational direction is the direction of the journal, then the eccentricity reaches a maximum.

4) The rotational speed of the revolution load from the mass eccentricity of a rotating system equals the journal speed. The revolution load with half of the journal speed results from different properties of the rotating system.

5) The half-frequency whirl is the result of a rotating load with specific frequencies on the radial sliding bearing; not the inherent attributes of the bearing.

\section{Conclusions}

1) The academic point that the half frequency whirl has nothing to do with the bearing load is wrong.

2) The half-frequency whirl should be redefined as the journal center whirl radius reaching a maximum for a radial sliding bearing while the rotating speed of the load is half the rotating speed of the journal.

3) The half-frequency whirl is the result of a rotating load with the rotational speed equal to half of the journal speed on the radial sliding bearing; not the inherent attributes of the bearing.

4) The presence of a revolution load and its rotational speed equal to half the speed of the journal are necessary conditions of the half-frequency whirl of the radial bearing. If there is no revolution load, then there is no half-frequency whirl. Engineering oil whip results from the vibration of the rotating system.

5) A precise radial sliding bearing lubrication theory should use cylindrical coordinates, consider the compressibility of the liquid, and consider the surface shear stress in the journal bearing capacity calculation.

\section{Acknowledgment}

The paper is supported by the National Natural Science Foundation of China (Grant No. 51374183 and 51490653).

\section{References}

Castro, H. F., Cavalca, K. L., \& Nordmann, R. (2008). Whirl and whip instabilities in rotor-bearing system considering a nonlinear force model. Journal of Sound and Vibration, 317, 273-293. http://dx.doi.org/10. 1016/j.jsv.2008.02.047

Dupont, R. (2003). On an isotropic and centrifugal force invariant layout of a conically shaped gas-lubricated high-speed spiral-groove bearing. Precision Engineering, 27, 346-361. http://dx.doi.org/10.1016/S01416359(03)00038-2

Kalita, M, \& Kakoty, S. K. (2004). Analysis of whirl speeds for rotor-bearing systems supported on fluid film bearings. Mechanical Systems and Signal Processing, 18, 1369-1380. http://dx.doi.org/10.1016/j.ymssp. 2003.09.002

Li, Z. (2008). Tubular mechanics in oil-gas wells and its applications (pp. 23-27). Beijing: Petroleum Industry Press. (in Chinese)

Li, Z., Li, J., Ma, X., \& Huang, W. (1995). Mathematical model of Newton liquid laminar flow induced by drillstring rotation and revolution. Mechanics and Practice, 17(5), 51-53. (in Chinese)

Liu, X., Zhang, X., \& Li, F. (2005). On the application of Newtonian viscous force formula. Journal of Mathematical Medicine, 18(4), 394-395. (in Chinese)

Newkirk, B. L. (1924). Shaft whipping. Gen. Electr. Rev., 27, 169-178.

Newkirk, B. L., \& Taylor, H. D. (1925). Shaft whipping due to oil action in journal bearings. Gen. Electr. Rev., $28,559-568$. 
Wen, S., \& Huang, P. (2008). Principles of tribology (3rd Ed., pp. 84-105). Beijing: Tsinghua University Press. (in Chinese)

Yao, F. (1991). Turbine shaft whirl and oil whip. Power Equipment, (10), 2-8. (in Chinese)

Yao, F. (1992). Half-speed whirl and oil whip. Power Equipment, (3), 3-9. (in Chinese)

Zhang, X., Duan, Z., \& Zhang, L. (2008). Study on oil whirl and oil oscillation of journal-bearing system. Journal of Taiyuan University of Technology, 39(3), 232-235. (in Chinese)

Zhou, Z., \& Tang, S. (2007). Minimum power consumption rate and vibrational principles in engineering mechanics (pp. 29-42). Beijing: Science Press. (in Chinese)

\section{Copyrights}

Copyright for this article is retained by the author(s), with first publication rights granted to the journal.

This is an open-access article distributed under the terms and conditions of the Creative Commons Attribution license (http://creativecommons.org/licenses/by/3.0/). 